\title{
Mit feinem Gespür
}

\section{Adrian Ritter}

Freier Journalist

\author{
Hochsensible Menschen sind schneller reizüberflutet, aber oft auch besonders \\ kreativ und empathisch. Wissenschaftlich ist das Konzept der Hochsensibilität um- \\ stritten. Für die Lebensqualität der Betroffenen aber kann es hilfreich sein. Eine \\ Spurensuche.
}

Für Marcel Suter (Name geändert) war es ein Schlüssel zum Verständnis. Als er vor einem Jahr das erste Mal den Begriff «Hochsensibilität» hörte, öffneten sich ihm Türen, um sich selber besser zu verstehen. Und um sein Leben zu ändern - denn er litt. Er war oft reizüberflutet, seit er nicht mehr freischaffend arbeitete und die für ihn nötigen Pausen in den Tagesablauf einbauen konnte. Sitzungen machten ihm Mühe, weil er sich im Gespräch nur schwer auf mehr als eine Person gleichzeitig konzentrieren kann. Oft spürte er abends sein mit Information überlastetes Gehirn regelrecht pochen.

Als ihn eines Tages jemand fragte, ob er hochsensibel sei, begann er nachzuforschen: «Ich war baff. Alles, was ich las, schien auf mich zuzutreffen.» So glaubte er auch plötzlich den Grund zu erkennen, warum er mehrmals Beziehungen beendet hatte: «Es wurde mir zu viel. Ich brauchte Raum und Zeit für mich.» Aufgrund der neuen Erkenntnis, vermutlich hochsensibel

\section{Hochsensible haben ein offeneres Filtersystem für äussere wie auch innere Reize.}

zu sein, war Marcel Suter bald klar, dass er seine Vollzeitanstellung kündigen würde. Heute hat er ein Teilzeitpensum und ist daneben wieder selbständig tätig. So kann er die nötigen Pausen wieder einplanen und Gruppensitzungen möglichst durch bilaterale Treffen ersetzen.

\section{Reizoffenes System}

Sie haben oft eine feine Wahrnehmungsgabe, ein reiches Innenleben, sind gewissenhaft, reflektiert und kreativ. Gleichzeitig sind sie schneller von Sinnesreizen überflutet als andere Menschen und brauchen mehr Rückzugsmöglichkeiten: Mit diesen Eigenschaften beschrieben die US-Psychologen Elaine N. Aron und Arthur Aron im Journal of Personality and Social Psychology 1997 erstmals die «highly sensitive person».
Im deutschsprachigen Raum hat sich dafür der Begriff der «Hochsensibilität» eingebürgert - auch wenn er bisweilen in Frage gestellt wird, weil er mit erlernter Feinfühligkeit verwechselt werden könnte. Das Psychologenpaar Aron meinte etwas anderes damit. In derselben Publikation prägten sie dafür auch den Begriff der "sensory processing sensitivity" - also der Sensitivität bei der Verarbeitung von Sinneswahrnehmungen.

Elaine N. Aron hat sich in den vergangenen zwanzig Jahren auf die Erforschung der Hochsensibilität spezialisiert. Sie spricht dabei von einer grundsätzlich höheren Aufnahme- und Verarbeitungsbereitschaft des neuronalen Systems. Anders ausgedrückt: Hochsensible haben ein offeneres Filtersystem für äussere wie auch innere Reize - von Sinneseindrücken bis zu den eigenen Gedanken.

Das Konzept der Hochsensibilität passt gemäss Aron $\mathrm{zu}$ einer Studie, die der russische Physiologe Iwan Pawlow in den 1920er Jahren durchgeführt hatte. Er setzte Probanden Lärm aus und untersuchte, ab wann bei ihnen Stressreaktionen auftreten. Das überraschende Ergebnis: Es ergab sich keine Normalverteilung, sondern eine Gruppe von Probanden unterschied sich markant vom Rest, indem sie deutlich früher mit Stress auf Lärm reagierte.

\section{Genetisch bedingt?}

Elaine N. Aron geht davon aus, dass eine «sensory processing sensitivity" in den meisten Fällen genetisch vererbt ist - ausser etwa, wenn sie aufgrund von Traumata entsteht. Hochsensibilität sei allerdings keine Krankheit oder Störung, sondern eine Variation des neuronalen Systems, schätzungsweise bei 15 bis 20 Prozent der Menschen zu finden - unabhängig von Geschlecht, Ethnie und Kultur. Hochsensibilität ist gemäss Aron bei allen höheren Tierarten mit etwa demselben Prozentsatz zu finden. Das mache evolutionsbiologisch auch Sinn: Ein Teil der Artgenossen ist besonders 


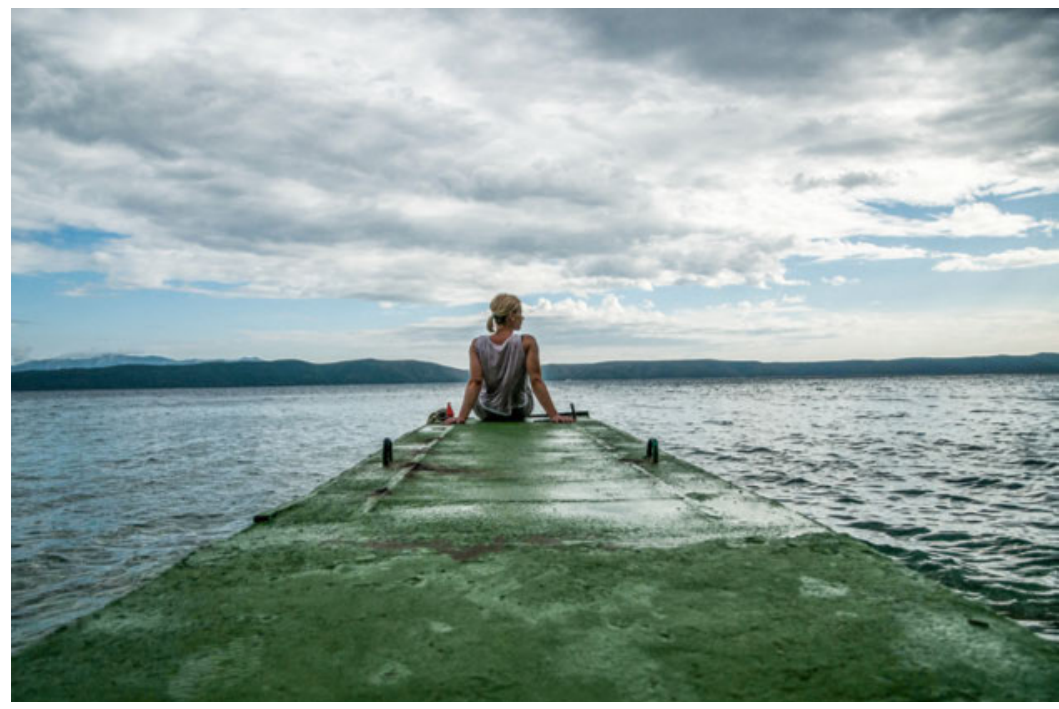

Ein gutes Gespür entwickeln, was einem gut tut und wo die eigenen Grenzen liegen. Hochsensible Menschen benötigen mehr Zeit zur Verarbeitung von Reizen und Rückzugsmöglichkeiten als andere Menschen.

wachsam etwa gegenüber Gefahren, aber auch mit Blick etwa auf neue Futterquellen.

Die Studie von 1997 blieb in der wissenschaftlichen Gemeinschaft nicht unwidersprochen. Einige kritisieren, der Test von Aron messe unterschiedliche Merkmale - einerseits die Neigung zu Gefühlen wie Angst oder Ärger und andererseits die Empfänglichkeit für neue Eindrücke. Der emeritierte Berliner Psychologieprofessor Jens Asendorpf betrachtet Hochsensibilität gar schlicht als Unterklasse von Neurotizismus. Elaine N. Aron betont umgekehrt, Hochsensibilität dürfe nicht mit Introversion oder Ängstlichkeit gleichgesetzt werden - auch Freude und Neugier könne die Erregung der Nerven bei Hochsensiblen hervorrufen. Ausserdem bedeute Neurotizismus, ohne ersichtlichen Grund ängstlich zu sein, was bei Hochsensiblen nicht der Fall sei.

\section{Doch eine Normalverteilung?}

«Hochsensibilität auf Neurotizismus zu reduzieren wird dem Phänomen nicht gerecht», ist auch Sandra Konrad überzeugt. Sie ist wissenschaftliche Mitarbeiterin an der Professur für Klinische Psychologie der Helmut-Schmidt-Universität in Hamburg und eine der ausgewiesensten Forscherinnen zu Hochsensibilität im deutschsprachigen Raum.

Konrad geht zwar ebenfalls davon aus, dass Hochsensibilität ein angeborener Wesenszug ist. Eine chinesische Studie habe kürzlich sogar bestimmte Gene identifiziert, die damit in Zusammenhang stehen könnten. Im Gegensatz zu Elaine N. Aron zweifelt Konrad allerdings daran, dass es sich bei den Hochsensiblen um eine separate, eindeutig unterscheidbare Gruppe handelt: «Ich denke, Sensibilität ist eher normalverteilt in der Gesellschaft.» Entsprechend sei es schwierig, klar zu definieren, wo Hochsensibilität beginnt. 15 bis 20 Prozent der Bevölkerung scheinen ihr denn auch etwas hoch gegriffen.

\section{Grosser Forschungsbedarf}

Das Grundproblem aus wissenschaftlicher Sicht: Hochsensibilität beruht bisher ausschliesslich auf Selbstzuschreibungen - anhand von Fragebogen. Einzelne MRI-Studien zeigen zwar eine stärkere Aktivierung im Gehirn von Menschen, die gemäss Fragebogen als hochsensibel gelten. «Es gibt aber bis jetzt keine anerkannten Kriterien zur objektiven Messung von Hochsensibilität. Der Forschungsbedarf ist auch sonst gross», sagt Sandra Konrad. Für sie stehen dabei folgende offenen Fragen im Vordergrund: eine grundlegende Theorie der Hochsensibilität, ein normierter Fragebogen und biologische Marker zur Identifikation von Hochsensibilität sowie die Klärung der Rolle von Genetik und Epigenetik im Zusammenhang mit Hochsensibilität.

\section{Das Leiden der Hochsensiblen}

Was die Genetik und Epigenetik anbelangt, ist noch ungenügend erforscht, inwiefern sich das Merkmal im Laufe des Lebens entwickelt: Einmal hochsensibel, immer hochsensibel? Oder lässt sich die Sensibilität beeinflussen? Diese Fragen sind umso wichtiger, als Hochsensible bisweilen unter ihrer höheren Empfindsamkeit leiden. Die Psychologin Christina Blach beschreibt in ihrer Dissertation, dass hochsensible Personen deutlich höhere Werte von Ängstlichkeit, Stress und Depressivität aufweisen. Auch Sandra Konrad sagt: «Mit der höheren Empfindsamkeit geht eine grössere Vulnerabilität für psychische Leiden einher.» Die Richtung der Kausalität sei allerdings zu wenig klar: Inwiefern führt mehr Angst zu grösserer Sensitivität - oder umgekehrt eine erhöhte Sensitivität zu mehr Ängstlichkeit?

Für Elaine N. Aron steht die zumeist angeborene Sensitivität am Anfang: Psychische Probleme entwickelten vor allem jene Hochsensiblen, die als Kinder unter belastenden Bedingungen aufwuchsen. Klar scheint aber auch, dass Hochsensible in unserer schnellebigen, lauten Gesellschaft nicht die einfachsten Lebensbedingungen vorfinden. So erstaunt es nicht, dass es für Hochsensible immer mehr spezifische Angebote gibt vom Coaching über die Achtsamkeitsmeditation bis zur Ernährungsberatung. Umgekehrt wären die Fähig- 
keiten der Hochsensiblen für unsere Gesellschaft aber wichtig, ist der Betriebsökonom Patrice Wyrsch überzeugt. Er widmet seine Dissertation an der Universität Bern der Neurosensitivität im Unternehmenskontext. Hochsensitive Menschen stellen für Unternehmen eine Bereicherung dar, indem sie etwa Chancen und Gefahren schneller erkennen, sagte Wyrsch kürzlich in einer Radiosendung.

\section{Hilfreiches Konzept}

Auch wenn Hochsensibilität als wissenschaftliches Konzept umstritten ist: Für Sandra Konrad ist klar, dass es Menschen gibt, die stärker auf Reize reagieren und entsprechend schneller reizüberflutet sind. Diesen Menschen könne das Konzept der Hochsensibilität helfen, sich selber besser zu verstehen und ihr Leben allenfalls anzupassen, um die Lebensqualität zu erhöhen: «Es geht vor allem darum, ein gutes Gespür dafür zu entwickeln, was einem gut tut und wo die eigenen Grenzen liegen. Oft heisst das auch: weniger Input, mehr Pause. So können Betroffene auch die Vorteile ihrer Hochsensibilität besser leben, etwa ihre zumeist ausgeprägte Intuition und Empathiefähigkeit», sagt Konrad.

\section{Die Vorteile leben}

Sandra Konrad rät Ärztinnen und Ärzten denn auch, das Thema anzusprechen, wenn bei Patienten das Leiden an einer möglichen Hochsensibilität im Vordergrund steht - und bei Bedarf etwa psychologische Fachhilfe vorzuschlagen. Ob die Patientinnen und $\mathrm{Pa}$ tienten mit dem Konzept der Hochsensibilität etwas anzufangen vermögen, wird sich im Gespräch zeigen.

\section{Was die Genetik und Epigenetik anbelangt, ist noch ungenügend erforscht, inwiefern sich die Hochsensibilität im Laufe des Lebens entwickelt.}

Der Autor dieses Artikels ist bei der Recherche immer wieder auf Menschen gestossen, die sich wie der eingangs erwähnte Marcel Suter im Konzept der Hochsensibilität wiedererkannt haben. Viele hatten ihr Leben schon zuvor intuitiv diesem Wesenszug angepasst - und dies nachher umso bewusster getan. «Früher zwang ich mich oft, über meine Grenzen hinauszugehen. Heute getraue ich mich eher zu sagen, ich brauche eine Pause», blickt Marcel Suter zurück. Dass der Aufenthalt in der Natur ihm gut tut, wusste er schon lange.

\section{Hochsensibilität und Medizin}

Ärztinnen und Ärzte werden in ihrer Tätigkeit vermutlich immer mal wieder Menschen begegnen, die man allenfalls als hochsensibel bezeichnen kann (vgl. Haupttext). Die US-Psychologin Elaine N. Aron - Begründerin des Konzepts der Hochsensibilität - macht in ihrer Publikation "Sind Sie hochsensibel?» (vgl. Literatur) bedenkenswerte Aussagen über Hochsensibilität und Medizin:

Hochsensible Menschen erscheinen in einem medizinischen Setting eher ängstlich und reagieren auf medizinische Untersuchungen und Behandlungen gewöhnlich mit starker nervlicher Erregung. Zudem reagierten Hochsensible sensibler auf Medikamente und seien meist schmerzempfindlicher. Gleichzeitig seien Hochsensible normalerweise sehr gewissenhaft - auch im Befolgen ärztlicher Anweisungen - und kooperativ, wenn es ihnen möglich ist.

Im besten Falle wüssten entsprechende Patienten um ihre Hochsensibilität und informierten ihren Arzt oder ihre Ärztin darüber. Dann können diese zusätzliche Massnahmen ergreifen, die Hochsensiblen den Arztbesuch leichter machen. Die Hochsensiblen wüssten idealerweise am besten selber, was hilfreich ist - zum Beispiel viele Erklärungen abgeben, ablenken oder im Gegenteil während eines Eingriffs eher schweigen, eine Vertrauensperson mitnehmen können, zusätzliche Beruhigungsmittel einnehmen oder sich beispielsweise vor einer invasiven Untersuchung mit dem entsprechenden Raum vertraut machen können. Gleichzeitig betont Elaine N. Aron dabei nochmals, dass die Hochsensibilität an sich keine Krankheit sei und somit keiner Behandlung bedürfe.

Monatelang war er schon alleine in der Wüste unterwegs. "Heute empfinde ich meine Hochsensibilität nicht mehr als störend, ich kann damit umgehen.» Dass er zum Beispiel kein Multitasking machen könne, habe er akzeptiert. Er könne sich dafür beispielsweise sehr lange auf eine Sache konzentrieren. «Manchmal wäre ich froh, ich hätte schon früher gewusst, dass ich hochsensibel bin. Ich hätte mich meinen Mitmenschen in gewissen Situationen besser verständlich machen können", sagt Marcel Suter.

\section{Bildnachweis}

(c) Eldarnurkovic| Dreamstime.com

\section{Literatur}

Elaine N. Aron: Sind Sie hochsensibel?, mvg Verlag, München, 2005 Online-Test zu Hochsensibilität (nach Elaine N. Aron): http://hsperson. com/test/highly-sensitive-test/

Spektrum der Wissenschaft (www.spektrum.de): Der Streit um die Feinfühligkeit (Artikel vom 9.6.2016)

Radiosendung zu Hochsensibilität vom 4.10.2017 auf SWR2 (www.swr.de) Informations- und Forschungsverbund Hochsensibilität (IFHS): www.hochsensibel.org

Jährlicher Kongress zu Hochsensibilität (Schweiz): www.hsp-kongress.ch/ Konrad, S., \& Herzberg, P. Y. (2017). Psychometric properties and validation of a German High Sensitive Person Scale (HSPS-G). European Journal of Psychological Assessment, 1-15

Christina Blach: Ein empirischer Zugang zum komplexen Phänomen der Hochsensibilität (Dissertation, 2016)
Korrespondenz: adrianritter[at]gmx.ch 